\title{
Nutilis Clear@ improves the safety and physiology of swallowing in patients with post-stroke oropharyngeal dysphagia
}

\author{
Mireia Bolivar-Prados \\ Laia Rofes \\ Viridiana Arreola \\ Sonia Guida \\ Weslania V. Nascimento \\ Alberto Martin \\ Natàlia Vilardell \\ Omar Ortega Fernández \\ Dina Ripken \\ Mirian Lansink \\ Pere Clavé
}

\section{Video Abstract}

Keywords: Neurogastroenterology and Motility, Universitat Autònoma de Barcelona, aspiration, deglutition disorders, shear viscosity, stroke, swallow response, thickener, xanthan gum, oropharyngeal dysphagia, Nutilis Clear, videofluoroscopy, kinematics, laryngeal vestibule closure, pharyngeal residue, unsafe swallow, bolus, esophagus, rheology, Penetration-Aspiration Scale

Posted Date: September 25th, 2020

DOI: https://doi.org/10.21203/rs.3.rs-83631/v1

License: (c) (i) This work is licensed under a Creative Commons Attribution 4.0 International License. Read Full License 


\section{Abstract}

Oropharyngeal dysphagia is characterized by difficulty swallowing and occurs in about $45 \%$ of stroke patients. Thickening agents are one effective way to help patients with dysphagia consume liquids and foods. However, few viscosity levels per study have been assessed, and the optimal levels for patients with oropharyngeal dysphagia have not yet been established. To address that gap, the Physiology Digestive team from the Hospital of Mataró recently tested Nutilis Clear ${ }^{\circledR}$, a xanthan gum-based thickener, at 7 shear viscosity levels. They looked at the effects of viscosity on the safety, efficacy, and physiology of swallowing in patients with post-stroke oropharyngeal dysphagia. The researchers first provided patients a thin liquid, followed by thickened boluses, from the highest to the lowest. Each bolus was provided in duplicate. Importantly, a safety rule was enforced: if a patient experienced aspiration with the first bolus of thin liquid, the second bolus was not administered, and the patient was advanced to the thickened boluses; if a patient experienced aspiration with any of the thickened boluses, the study was terminated for that patient. For videofluoroscopic analysis, the team prepared the boluses with a watersoluble X-ray contrast solution. The study enrolled 120 patients with post-stroke oropharyngeal dysphagia, and the data for 114 patients was analyzed. The excluded patients did not receive the thickened boluses or showed protocol deviations. The results for the two groups were comparable. Overall, the team found that the gum-based thickener positively affects three aspects of swallowing for patients with post-stroke oropharyngeal dysphagia: safety, defined as the percentage of patients with safe swallowing; efficacy; and physiology kinematics. Nearly $60 \%$ of the patients presented unsafe swallow with the thin liquid. However, when the bolus was thickened with Nutilis Clear to viscosities ranging from 150 to $2000 \mathrm{mPa} \cdot \mathrm{s}$, the percentage of patients with safe swallowing significantly increased, with unsafe swallow reduced to less than $10 \%$ at the higher viscosities. Pharyngeal residue did not increase significantly relative to the thin liquid for any of the thickened viscosities. Additionally, the time required for the laryngeal vestibule to close after initiating a swallow significantly decreased at all viscosities versus the thin liquid. A therapeutic range of 150 to $800 \mathrm{mPa} \cdot \mathrm{s}$. was defined: 150 was the lowest viscosity to have a significant effect on the safety of swallowing, and 800 presented a similar level of protection relative to higher viscosities. Nutilis Clear increases swallowing safety in a viscositydependent manner, without increasing pharyngeal residue. In addition, the reduction in time to laryngeal vestibule closure caused by the thickening agent suggests a mode of action beyond a simple "compensatory" effect. These results confirm the effectiveness of this specific xanthan gum-based thickener and support the recommendation of use in dysphagia management in stroke patients. 